\title{
REMOTE POINTS IN $\beta R$
}

\author{
N. J. FINE ${ }^{1,2}$ AND L. GILLMAN ${ }^{1,8}$
}

0. Summary. A construction by now well known establishes the existence of a point in $\beta R$ that is not in the closure of any $R$-closed discrete subset of $\mathbf{R .}^{4}$ Our purpose is to establish the stronger conclusion:

(I) There exists a point $p$ in $\beta R$ that is not in the closure of any discrete subset of $\mathbf{R}$;

equivalently (as shown below):

(II) There exists a $z$-ultrafilter $\boldsymbol{A}^{p}$ on $\mathbf{R}$ no member of which is nowhere dense.

The proof turns out to be considerably more difficult than anticipated. If we assume the continuum hypothesis (designated $[\mathrm{CH}]$ ), then we can find such a point $p(2.5)$; however, we do not know whether the continuum hypothesis is necessary. The result is obtained as a consequence of a more general theorem: for a suitably restricted class of spaces $X$, a point with a somewhat stronger property exists if and only if $X$ admits an unbounded continuous function (2.3).

A byproduct is: $[\mathrm{CH}]$ there exists a countable, completely regular space without isolated points, one of whose points is not a limit point of any discrete set (2.6).

1. Preliminaries. We recall that a subset $S$ of a space $X$ is nowhere dense if $\mathrm{cl} S$ has void interior. Discrete subset means discrete in the relative topology. $\mathbf{R}$ denotes the real line, $\mathbf{Q}$ the subspace of rationals, $\mathbf{N}$ the subspace of natural numbers.

1.1. Proposition. Given $F$ nowhere dense and closed in $\mathbf{R}$, there exists a discrete subset $D$ of $\mathbf{R}$ such that $D \cap F=\varnothing$ and $D \cup F=\mathrm{cl}_{\mathbf{R}} D$.

Proof. $X-F$ is a union of disjoint open intervals $I_{\alpha}$. Choose

Received by the editors January 7, 1961.

1 This paper was written while both authors were members of the Institute for Advanced Study.

2 This research was supported by Air Force Contract AF 18 (603)-65.

- National Science Foundation Senior Post-Doctoral fellow.

4 As far as we know, the result and the proof reproduced here (1.3) are due to W. F. Eberlein; they are credited to him in M. Henriksen's Wisconsin thesis, 1951. 
$D_{\alpha} \subset I_{\alpha}$ discrete and with the endpoints of $I_{\alpha}$ as its only limit points. Put $D=\mathrm{U}_{\alpha} D_{\alpha}$.

1.2. All spaces considered will be assumed to be completely regular. $\boldsymbol{Z}(f)$ denotes the zero-set $\{x \in X: f(x)=0\}, f$ being a continuous real-valued function on $X$. In $\mathbf{R}$ (or any metric space), the zero-sets are just the closed sets, but in general, they form a proper subclass. A z-filter is a family of zero-sets closed under finite intersection, containing with each member all larger zero-sets, and not containing $\varnothing$; a $z$-ultrafilter is a maximal $z$-filter.

The $z$-ultrafilters on $X$ are indexed by the points of $\beta X$ (the StoneCech compactification of $X)$; this correspondence, $p \rightarrow A^{p}(p \in \beta X)$, is one-one and satisfies

$$
Z \in A^{p} \text { if and only if } p \in \operatorname{cl}_{\beta x} Z,
$$

where $Z$ is a zero-set $[1,6.5]$. A $z$-ultrafilter $A^{p}$ is free if $\cap A^{p}$ is empty $(p \in \beta X-X)$.

The equivalence of (I) with (II) follows from 1.1 and (1).

1.3. Theorem. ${ }^{4}$ There exists a $z$-ultrafilter $\boldsymbol{A}^{p}$ on $\mathbf{R}$ all of whose members have infinite Lebesgue measure. In particular, if $S$ is a closed discrete subset of $\mathbf{R}$, then $p \notin \mathrm{cl}_{\beta \mathbf{R}} S$.

Proof. Let $F$ denote the $z$-filter of all closed sets in $\mathbf{R}$ whose complements have finite measure. Let $A^{p}$ be any $z$-ultrafilter containing $\mathcal{F}$. If $Z$ is any closed set of finite measure, then some open set $U \supset Z$ has finite measure, whence $\mathrm{R}-U \in \mathcal{F} \subset A^{p}$; since $Z$ is disjoint from $\mathbf{R}-U, Z \notin A^{p}$.

1.4. In contrast, we can find a discrete $D$ for which $p \in \operatorname{cl}_{\beta \mathrm{R}} D$. Let $F$ be any nowhere dense closed set whose complement is of finite measure; then $F \in \mathcal{F} \subset A^{p}$, so that $p \in \mathrm{cl}_{\beta \mathrm{R}} F$. Choose a discrete set $D$ such that $\operatorname{cl}_{\mathbf{R}} D \supset F(1.1)$; then $p \in \operatorname{cl}_{\beta \mathbf{R}} \operatorname{cl}_{\mathbf{R}} D=\operatorname{cl}_{\beta \mathbf{R}} D$.

Let $\mu$ denote Lebesgue measure on $\mathbf{R}$. To find a $z$-ultrafilter containing no nowhere dense set, one might naturally begin by considering the family $\mathcal{E}$ of all closed sets $Z$ in $\mathbf{R}$ for which $\mu$ (int $\operatorname{cl}(\mathbf{R}-Z)$ ) is finite. Since $\mu(U) \leqq \mu($ int $\mathrm{cl} U)$ for open $U, \varepsilon$ is a subfamily of Eberlein's z-filter $\mathcal{F}$; therefore $\mathcal{E}$ is contained in a z-ultrafilter. However, we shall exhibit two members of $\varepsilon$ whose intersection is nowhere dense; since this intersection must belong to any $z$-filter containing $\mathcal{E}$, the attempted construction breaks down.

Let $N$ be a nowhere dense closed set whose complement is of finite measure and such that the family of disjoint open intervals in the canonical decomposition of $\mathrm{R}-N$ is ordered, in its natural order, 
like the set of rationals. Choose complementary, dense subfamilies of these intervals, and let $A_{0}$ and $B_{0}$ denote their unions. Every point of $N$ belongs to $\mathrm{cl} A_{0} \cap \mathrm{cl} B_{0}$. Define $A=A_{0} \cup N$ and $B=B_{0} \cup N$; then $A$ and $B$ are closed. Since

$$
\mu(\text { int } \mathrm{cl}(R-A))=\mu\left(\text { int } \mathrm{cl} B_{0}\right)=\mu(\text { int } B)=\mu\left(B_{0}\right)<\infty,
$$

$A \in \mathcal{E}$. Likewise, $B \in \mathcal{E}$. But $A \cap B$ is the nowhere dense set $N$.

Notice, by the way, that $\mu($ int $A)+\mu($ int $B)<\infty$, while $\mu(\operatorname{int}(A \cup B))$ $=\infty$-in fact, $A \cup B=\mathbf{R}$.

\section{The main results.}

2.1. Lemma. Given a space $X$ and a nonnegative, unbounded, continuous function $h$ on $X$, consider any unbounded increasing sequence of positive values of $h$, and choose a sequence $\left(J_{n}\right)_{n<\omega}$ of disjoint closed intervals of $\mathbf{R}$ each of which contains one of these numbers in its interior. Then the sets

$$
E_{n}=\left\{x \in X: h(x) \in J_{n}\right\}
$$

satisfy:

(i) Each $E_{n}$ is a zero-set with nonvoid interior.

(ii) If $\left(F_{n}\right)$ is any sequence of zero-sets in $X$, with $F_{n} \subset E_{n}$, then $U_{n} F_{n}$ is a zero-set.

Proof. (i) is obvious: since $J_{n}$ is a zero-set (in $\mathbf{R}$ ) with nonvoid interior, its total preimage $E_{n}$ under $h$ is a zero-set (in $X$ ) with nonvoid interior.

To prove (ii), we argue in more detail. For each $n$, there exists a continuous function $s_{n}$ on $\mathbf{R}$ equal to 1 precisely on the closed interval $J_{n}$, equal to 0 precisely on the disjoint closed set $\bigcup_{k \neq n} J_{k}$, and assuming intermediate values everywhere else. Given $\left(F_{n}\right)$ as in (ii), write $F_{n}=\boldsymbol{Z}\left(f_{n}\right)$, with $0 \leqq f_{n} \leqq 2^{-n}$. Consider the infinite sum

$$
g=f_{0} \cdot\left(s_{0} \circ h\right)+f_{1} \cdot\left(s_{1} \circ h\right)+\cdots \cdot
$$

Because of uniform convergence, this defines $g$ as a continuous function on $X$. Clearly, $g$ has no zeros outside $U_{n} E_{n}$; and for each $n, g$ agrees with $f_{n}$ on $E_{n}$. Therefore, $\bigcup_{n} F_{n}$ is the zero-set $\boldsymbol{Z}(g)$.

2.2. A space $X$ is pseudocompact if every continuous real-valued function on $X$ is bounded; this is the case if and only if every free $z$-ultrafilter is closed under countable intersection [1, 5.8 and 5.14]. A cardinal $m$ is measurable if there exists a nontrivial $\{0,1\}$-valued measure (countably additive) on a set of cardinal $\mathfrak{m}$. Each such measure determines a free ultrafilter closed under countable intersection, and conversely, the members of the ultrafilter being the sets 
of measure 1. Every cardinal greater than a measurable cardinal is measurable. Every cardinal smaller than the first strongly inaccessible cardinal is nonmeasurable. [1, Chapter 12].

2.3. Theorem. Let $X$ be a space such that:

(a) The cardinal of the set of all isolated points is nonmeasurable.

(b) There exists a family $\mathfrak{u}$ of $\aleph_{1}$ dense open sets such that every dense open set contains a member of $\mathcal{u}$.

Then a necessary and sufficient condition that $X$ not be pseudocompact is that there exist a free z-ultrafilter $A^{p}$ on $X$ such that every dense open set contains a member of $\boldsymbol{A}^{p}$.

Proof. Necessity. By hypothesis, $X$ admits an unbounded continuous function $h$, which we may assume to be nonnegative. For this $h$, let $E_{n}$ be as in (2), and define

$$
S_{n}=\bigcup_{k \geqq n} E_{k}
$$

By Lemma 2.1, each $S_{n}$ is a zero-set with nonvoid interior. Notice that

$$
\text { int } S_{n}=\underset{k \geq n}{\bigcup} \text { int } E_{k} .
$$

Write $\mathcal{U}=\left(U_{\alpha}\right)_{\omega \leqq \alpha<\omega_{1}}$. Consider any ordinal $\alpha$, with $\omega \leqq \alpha<\omega_{1}$, and assume inductively that for all $\xi<\alpha$, we have defined a zero-set $S_{\xi}$ such that the family (int $\left.S_{\xi}\right)_{\xi<\alpha}$ has the finite intersection property.

To define $S_{\alpha}$, we first rearrange this countable family (int $S_{\xi}$ ) $\varepsilon<\alpha$ into an ordinary sequence $\left(T_{n}\right)_{n<\omega}$. Next, we define an increasing sequence $\left(n_{i}\right)$ of finite ordinals and a sequence $\left(F_{j}\right)$ of zero-sets, as follows. Let $k<\omega$, and assume inductively that $n_{i}$ have been defined for all $i<k$, with $n_{0}<\ldots<n_{k-1}$, and that $F_{j}$ have been defined for all $j \leqq n_{k-1}$. By assumption, $T_{0} \cap \cdots \cap T_{k}$ meets int $S_{n_{k-1}+1}$ (where $\left.n_{-1}=-1\right)$ and hence meets int $E_{n_{k}}$ for some $n_{k}>n_{k-1}$. Since $U_{\alpha}$ is open and dense, we can choose a zero-set $F_{n_{k}}$ with nonvoid interior and satisfying

$$
F_{n_{k}} \subset T_{0} \cap \cdots \cap T_{k} \cap E_{n_{k}} \cap U_{\alpha} .
$$

Finally, we put $F_{j}=\varnothing$ for $n_{k-1}<j<n_{k}$.

This completes the definition of the sequence $\left(F_{j}\right)$. Obviously, $F_{j} \subset E_{j}$ for each $j$. We now define

$$
S_{\alpha}=F_{0} \cup F_{1} \cup \cdots \text {. }
$$

By the lemma, $S_{\alpha}$ is a zero-set. To complete the induction step, we observe that any finite intersection of sets int $S_{\xi}$, for $\xi<\alpha$, contains 
a set $T_{0} \cap \cdots \cap T_{k}$; therefore it contains $F_{n_{k}}$ and hence meets int $S_{\alpha}$. Consequently, the collection (int $\left.S_{\xi}\right)_{\xi \leq \alpha}$ has the finite intersection property.

The family of zero-sets $S_{\alpha}\left(\alpha<\omega_{1}\right)$ thus defined has the finite intersection property, and, accordingly, it is contained in a $z$-ultrafilter $\boldsymbol{A}^{p}$. Since $\bigcap_{n<\omega} S_{n}=\varnothing, \boldsymbol{A}^{p}$ is free. By (4) and (5), $U_{\alpha} \supset S_{\alpha}$; it follows that every dense open set contains a member of $A^{p}$.

This completes the proof of necessity. We remark for later reference that by (1),

$$
p \in \operatorname{cl}_{\beta X} S_{0}
$$

Sufficiency. We shall prove that the given $z$-ultrafilter $\boldsymbol{A}^{p}$ is not closed under countable intersection. We assume the contrary and obtain a contradiction.

With (b), our hypothesis implies that $A^{p}$ has a subfamily $\left(Z_{\alpha}\right)_{\alpha<\omega_{1}}$ such that every dense open set contains some $Z_{\alpha}$.

First we observe that each $A \in A^{p}$ contains a nonisolated interior point. For, if not, then int $A$ consists entirely of isolated points of $X$. Since $A$-int $A$ is closed and nowhere dense, its complement contains some $Z_{\alpha}$; thus, int $A \cap Z_{\alpha}=A \cap Z_{\alpha} \in A^{p}$. The trace of $A^{p}$ on int $A \cap Z_{\alpha}$ is then a free ultrafilter closed under countable intersection; but by (a), this is not possible.

We proceed with the proof. Given $\alpha<\omega_{1}$, assume, inductively, that zero-sets $A_{\xi} \in A^{p}$ have been defined for all $\xi<\alpha$ such that

$$
A_{\xi} \subset Z_{\xi}
$$

for $\xi<\alpha$ and

$$
A_{\xi} \supset A_{\xi+1}
$$

for $\xi+1<\alpha$, and that points $x_{\xi}$ have been defined for all $\xi+1<\alpha$ such that

$$
x_{\xi} \text { is a nonisolated interior point of } A_{\xi}-A_{\xi+1}
$$

for $\xi+1<\alpha$.

If $\alpha$ is a limit ordinal or zero, define

$$
A_{\alpha}=\bigcap_{\xi<\alpha} A_{\xi} \cap Z_{\alpha}
$$

by our assumption, $A_{\alpha} \in A^{p}$.

If $\alpha-1$ exists, choose a nonisolated interior point $x_{\alpha-1}$ of $A_{\alpha-1}$. Since $A^{p}$ is free, there exists $Z \in A^{p}$ for which $x_{\alpha-1} \notin Z$. Define 


$$
A_{\alpha}=Z \cap A_{\alpha-1} \cap Z_{\alpha}
$$

Then $x_{\alpha-1}$ is a nonisolated interior point of $A_{\alpha-1}-A_{\alpha}$.

By induction, we have defined $A_{\xi}$ and $x_{\xi}$ such that (7), (8), and (9) hold, for all $\xi<\omega_{1}$. The latter two conditions imply that the set $D$ of all points $x_{\xi}$ is nowhere dense; hence $X-\operatorname{cl} D$ contains some $Z_{\xi}$. But this is impossible, since $x_{\xi} \in D$, while by (7) and (9), $x_{\xi} \in Z_{\xi}$. This concludes the proof of the theorem.

2.4. It is clear that portions of the result can be stated more generally. For example, hypothesis (a) was not used in the proof of necessity.

It is also possible to extract from the proof some finer information contrasting closed sets with zero-sets. The relevant observation is that a nowhere dense closed set in $X$ may have a point $p$ of $\beta X$ in its closure even though no nowhere dense zero-set has; e.g., consider the set of limit ordinals in the space of all countable ordinals (see $[1,5.12])$.

We remark that condition (b) is properly weaker than the requirement that $X$ admit at most $\aleph_{1}$ dense open sets; for example, the prefilter of dense open subsets of $\beta \mathbf{N}$ is of cardinal $\geqq \exp \exp \boldsymbol{\aleph}_{0}>\boldsymbol{\aleph}_{1}$, but it has a base-namely, $\{\mathbf{N}\}$-of cardinal 1 .

2.5. TheOREM. [C.H]. There exists a point $p$ in $\beta \mathrm{R}$ that is not in the closure of any discrete subset of $\mathbf{R}$. In fact, the set of all such points is dense in the space $\beta \mathbf{R}-\mathbf{R}$.

Proof. Under [CH], $\mathbf{R}$ has just $\boldsymbol{\aleph}_{1}$ dense open sets; also, $\mathbf{R}$ is not pseudocompact. By Theorem 2.3 (necessity), there exists a free $z$ ultrafilter $\boldsymbol{A}^{p}$ on $\mathbf{R}$ such that every dense open set contains a member of $\boldsymbol{A}^{p}$; hence no member of $\boldsymbol{A}^{p}$ can be nowhere dense. As observed at the close of 1.2 , this is equivalent (for the space $R$ ) to the first assertion of the corollary.

We wish to show now that if $q$ is any point of $\beta \mathbf{R}-\mathbf{R}$ and $V$ is any closed neighborhood of $q$ in $\beta \mathbf{R}$, then at least one such point $p$ lies in $V$. Define $f(x)=x$ for $x \in \mathbf{R}$, and choose a nonnegative continuous function $g$ on $\beta R$ equal to 1 at $q$ and equal to 0 everywhere on $\beta R-V$. Next, define

$$
h=f \cdot(g \mid \mathbf{R}) \text {; }
$$

then $h$ is nonnegative, unbounded, and continuous (on R). Clearly, for this $h$, the sets $E_{n}$ of (2) are contained in $V$. So, then, is their union, $S_{0}$ (see (3)). According to (6), the point $p$ constructed in the ensuing proof lies in $\mathrm{cl}_{\beta \mathbf{R}} S_{0}$. Since $V$ is closed, $p \in V$. This concludes the proof. 
Similarly, $[\mathrm{CH}]$ there exists a point $p^{\prime}$ of $\beta Q$ that is not in the closure of any discrete subset of $\mathbf{Q}$.

2.6. Corollary. [CH]. There exists a countable space without isolated points, one of whose points is not a limit point of any discrete set.

Proof. $Q \cup\{p\}$ and $Q \cup\left\{p^{\prime}\right\}$, where $p$ and $p^{\prime}$ are as above, are such spaces.

3. Pseudocompact spaces. A space $X$ is pseudocompact if and only if every nonempty zero-set in $\beta X$ meets $X$. When, in contrast, every point of $\beta X-X$ is contained in a zero-set of $\beta X$ disjoint from $X$, then $X$ is said to be realcompact [1, Chapter 8].

3.1. Theorem. Let $S$ be a realcompact space, let $H \subset \beta S-S$, and define $X=S \cup H$.

If $X$ is pseudocompact, then $H$ is dense in $\beta S-S$.

Conversely, if $H$ is dense in $\beta S-S$, and if, in addition, $S$ is locally compact, then $X$ is pseudocompact.

Proof. If $H$ is not dense, there exists a nonempty open set $U$ in $\beta S-S$ disjoint from $H$. Write $U=V-S$, where $V$ is open in $\beta S$. Pick $p \in U$. There exists a zero-set $Z$ in $\beta S$ such that $p \in Z \subset V$. Since $V \cap H=\varnothing$, we have $Z \cap H=\varnothing$. Since $S$ is realcompact, there is a zero-set $Z^{\prime}$ in $\beta S$ such that $p \in Z^{\prime} \subset \beta S-S$. Then

$$
p \in Z \cap Z^{\prime} \subset(\beta S-H-S)=\beta S-X=\beta X-X .
$$

Therefore $X$ is not pseudocompact.

Conversely, if $X$ is not pseudocompact, there exists a continuous function $f$ on $\beta X$ whose zero-set is nonempty and is contained in $\beta S-X \subset \beta S-S$. If $S$ is locally compact as well as realcompact, then $\boldsymbol{Z}(f)$ has nonvoid interior in $\beta S-S[2,3.1]$. Since $H$ does not meet $\boldsymbol{Z}(f), H$ is not dense.

3.2. ExAmples. For $H \subset \beta \mathbf{R}-\mathbf{R}, \mathbf{R} \cup H$ is pseudocompact if and only if $H$ is dense in $\beta \mathbf{R}-\mathbf{R}$. For $H \subset \beta \mathbf{N}-\mathbf{N}, \mathbf{N} \cup H$ is pseudocompact if and only if $H$ is dense in $\beta \mathbf{N}-\mathbf{N}$.

Local compactness, assumed in the second half of the theorem, is critical. Let $f(x)=x$ on the space $\mathbf{Q}$, and let $X$ denote the set of all points of $\beta Q$ to which $f$ has a continuous extension. Clearly, $X$ is not pseudocompact. But the set $H=X-\mathbf{Q}$ is dense in $\beta \mathbf{Q}-\mathbf{Q}$. For, consider any point $q$ of $\beta Q-Q$ and any closed neighborhood $V$ of $q$ in $\beta \mathbf{Q}$. Then $V \cap Q$ contains a bounded interval $J$ of $\mathbf{Q}$; and $\operatorname{cl}_{\beta \mathbf{Q}} J \subset V$. Obviously, cl $J$ is nonempty; and since $f$ is bounded on $J, \mathrm{cl} J-J \subset H$. So $H$ is dense. 
3.3. Let $\Gamma$ denote the set of all points of $\beta R-R$ that are not in the closure of any discrete subset of $R$, and let $\Delta$ denote the complementary subset of $\beta \mathbf{R}-\mathbf{R}$.

THEOREM. Both $\Delta$ and $[\mathrm{CH}] \Gamma$ are dense in $\beta \mathbf{R}-\mathbf{R}$. Equivalently: both $\mathrm{R} \cup \Delta$ and $[\mathrm{CH}] \mathrm{R} \cup \Gamma$ are pseudocompact spaces.

Proof. The equivalence has just been noted. Since any closed neighborhood in $\beta R$ of a point of $\beta R-R$ contains an unbounded discrete subset of $\mathbf{R}, \mathbf{R} \cup \Delta$ is dense. That $[\mathrm{CH}] \mathbf{R} \cup \Gamma$ is dense was established in Theorem 2.5.

\section{REFERENCES}

1. L. Gillman and M. Jerison, Rings of continuous functions, Van Nostrand, Princeton, N. J., 1960.

2. N. J. Fine and L. Gillman, Extension of continuous functions in $\beta \mathrm{N}$, Bull. Amer. Math. Soc. 66 (1960), 376-381.

University of Pennsyluania and

UNIVERSITY OF ROCHESTER 